\title{
Analytical solutions of velocity profile in flow through submerged vegetation with variable frontal width
}

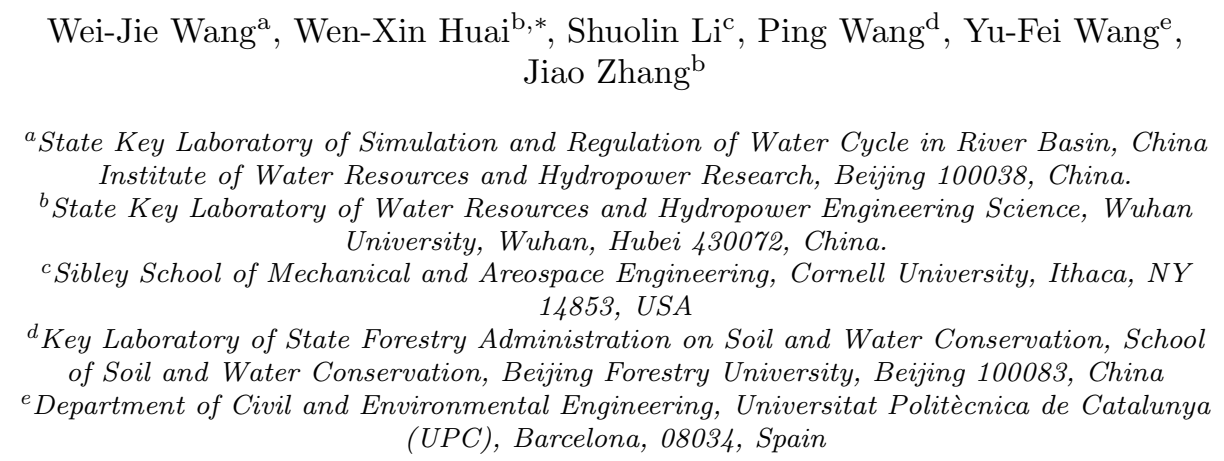

\begin{abstract}
Flow within vegetation is one of the main driving forces for material exchange and energy transfer in wetland systems. Impacted by vegetation, the flow velocity profile illustrates distortions to the classic logarithmic velocity profile and has attracted much attention among researchers. Different from analytical models of velocity distribution in literature, which is mainly suitable for vegetation with uniform frontal width, this paper establishes new analytical solutions of the velocity profile for vegetation such as shrub and sedge that have a variable frontal width in the vertical direction. A new shape function is proposed under these conditions in which the frontal width exhibits a gradual increase in the vertical direction from bottom up in the vegetation. Along with different closure models for eddy viscosity in the vegetation layer and surface layer, analytical solutions of the velocity profile are derived from the momentum equations. Good agreement between calculated and measured data shows
\end{abstract}

\footnotetext{
* Corresponding author

Email address: wxhuai@whu.edu.cn (Wen-Xin Huai)
} 
our analytical model is effective in predicting velocity profiles.

Keywords: Analytical solution; Velocity profile; Vegetation; Turbulent flow;

Flow resistance.

\section{Introduction}

Human activities along with the rapid development of society and industrialization have unleashed huge pressure on aquatic ecosystems that include not only the artificial modification of rivers such as projects adopting dams to intercept rivers, cutting and straightening winding rivers, hardening of side slopes and channel bottoms with concrete, but also waste water pollution from agricultural and industrial production. Focusing on these problems, ecological restoration aims to repair damaged water systems and to rebuild healthy aquatic ecosystems to provide a sustainable and healthy development of ecosystems $(\mathrm{Li}$ et al., 2015, Yu and Wang, 2014, Mi et al., 2015).

Vegetation is widely used in river and wetland water ecological treatment and restoration (Constança Aguiar, F. et al. 2011; Stromberg, 2001) because it serves many eco-functions, such as fixation through roots for maintaining riverbed stability, water purification through the absorption capacity of the epidermis, and enriching biodiversity features by providing attachment matrices and habitats for organisms.

All of these ecological restoration measures require a description of flow through vegetation, where the flow region is partitioned into different layers according to the dominant vortical structures, resulting in a complex flow pattern (Nepf, 1999, Nepf and Ghisalberti, 2008, Poggi et al., 2004a). How to calculate the flow velocity is a key problem and a fundamental research topic for furthering the studies of contaminant transport and energy loss features. Flow distributions are calculated with two main approaches: numerical models and analytical models. Numerical models, such as direct numerical simulation (DNS), large-eddy simulation (LES), and many others, mainly focus on the local velocity and turbulent features. They yield relatively accurate results 
by massive calculations with suitable parameters (Lu and Dai, 2016, Shimizu et al. 1991; Stoesser et al., 2009, Zhang et al. 2013). Sometimes we do not need to focus on each point in the flow region but instead care more about the planar-averaged velocity or bulk velocity for a preliminary prediction. In such instances, an analytical solution with less calculation arises that reveals the principle or bulk law for flow through vegetation. For simplicity, vegetation fan always be represented as cylinders or strips (Klopstra et al., 1996, Shimizu et al., 1991; Kouwen et al., 1969, Nepf and Ghisalberti, 2008; Huai et al., 2009, Wang et al. 2018b), and the flow region can be divided into different layers depending on the turbulence model adopted: (1) The one-layer model takes the flow region as a whole, and the friction factor scales with the ratio of vegetation height to flow depth (Cheng, 2015) or scales with the ratio of vegetation-related roughness height to vegetation-related hydraulic radius (Wang et al., 2018c). Interestingly, both scale parameters are around 3/2. (2) In the two-layer model, the flow region is divided into a vegetation layer and a surface layer (Yang and Choi 2010, Huai et al. 2013), and different momentum equations are proposed for each layer. For rigid dense vegetation, the flow velocity is assumed to be uniform in the vegetation layer and is derived from the momentum balance between the vegetation drag and the gravity component(Yang and Choi, 2010, Baptist et al. 2007). Furthermore, the velocity at the surface layer is expressed by a logarithmic formula with zero-plane displacement(Thom, 1971). For flexible vegetation, the bending of the stem was considered by Huai et al. (2013) with two kinds of resistance force, specifically, drag force and friction force. These forces scale with the square of the flow velocity, and an analytical solution was proposed by solving the momentum equation in each layer. Further investigation showed that a linear drag-velocity relation occurs when bending is very large (Wang et al., 2015b). (3) In the multiple-layer model, a sub-layer occupying a very thin layer near the bottom of the channel is separated from the vegetation layer (Baptist et al. 2007), and an additional layer called the mixing layer, which is separated from both the vegetation layer and the surface layer near the top of the vegetation (Okamoto and Nezu, 2009, Katul et al., 2002, 
Nepf, 2012, Ghisalberti and Nepf, 2002), where the flow features are related to the frequency of the Kelvin-Helmholtz instability (Peters, 2012). Moreover, vegetation in this research has the same height, whereas vegetation in nature is always non-uniform in height. Research was conducted on this topic (Huai et al. 2014, Liu et al., 2010), in which a combination of the aforementioned two-, three- or multiple-layer model was adopted and improved for different vegetation heights.

In some manner, a solution of the velocity profile is adopted for determining the resistance of the vegetated channel when solving the Saint-Venant equations (SVEs) describing water flow. As is well-known, there is a need for the closure of the energy slope $S_{f}$, for the flow, which may be derived from the force balance with a 'local uniform' assumption (Thompson et al., 2011, Wang et al., 2015a, 2018a). When dealing with open channel flow without obstacles, the classic Manning formula is adopted,

$$
S_{f}=\left(\frac{2 g n^{2}}{R^{4 / 3}}\right) \frac{U_{b}^{2}}{2 g},
$$

where $g$ is acceleration due to gravity, $n$ Manning's coefficient of roughness, $U_{b}$ the bulk velocity for whole flow depth, and $R$ the hydraulic radius. For flow within the vegetation, the energy slope $S_{f}$ is complicated and may be derived from a force balance equation. Our previous work (Wang et al., 2015a) derived an equation for the flow through emergent vegetation for a given length-scale $d x$ along the streamwise direction,

$$
\gamma B h_{w} d x(1-\phi) S_{f}=B d x F_{d, b u l k}+B d x(1-\phi) \tau_{\text {ground }}+2 h_{w} d x \tau_{w a l l}
$$

where $\gamma$ denotes the bulk density of water, $B$ channel width, $h_{w}$ flow depth, $\phi$ the area concentration of vegetation stems, $F_{d, b u l k}$ the vegetation form drag per unit ground area, $\tau_{\text {ground }}$ friction along the bottom of the channel, and $\tau_{\text {wall }}$ friction along the sidewall of the channel. For flow through vegetation, friction along the sidewall and bottom can always be ignored Wang et al. (2015a), resulting in a balance between the streamwise gravity and form drag of vegetation as

$$
\gamma h_{w}(1-\phi) S_{f}=F_{d, b u l k}
$$


where the form drag acting on the unit ground area is calculated from

$$
F_{d, b u l k}=C_{d} A_{\text {frontal }} \frac{\rho U_{b}^{2}}{2},
$$

with $C_{d}$ the drag coefficient and $A_{\text {frontal }}$ the total frontal area of vegetation stems per unit bed ground, obtained using

$$
A_{\text {frontal }}=m D h_{w},
$$

where $m$ is the vegetation density, denoting the number of vegetation stems per unit bed ground, and $D$ the frontal width for a single stem. The bulk form drag in equation 3 then becomes

$$
F_{d, b u l k}=C_{d} m D h_{w} \frac{\rho U_{b}^{2}}{2} .
$$

For submerged vegetation, the flow region is divided into two layers (vegetation layer and surface layer). The drag in the vegetation layer is expressed by

$$
F_{d, \text { bulk }}=C_{d} m D h_{v} \frac{\rho U_{v}^{2}}{2},
$$

where $h_{v}$ is the vegetation height and $U_{v}$ the depth-averaged velocity for the vegetation layer.

The closure of $S_{f}$ for emergent vegetation in flow is then

$$
S_{f}=\left(\frac{C_{d} m D}{1-\phi}\right) \frac{U_{b}^{2}}{2 g},
$$

and for submerged vegetation is

$$
S_{f}=\left[\frac{C_{d} m D h_{v}}{(1-\phi) h_{w}}\right] \frac{U_{v}^{2}}{2 g} .
$$

All considered, the velocity profile is key for the description of fluid motion. However, several analytical models exist that have been derived for simplified vegetation models, i.e., a cylinder or single strip with a constant frontal width limited by the solvability of the momentum equation. Different from existing studies on analytical models of velocity distribution with a uniform frontal width of vegetation, the main innovative points of this paper are: (1) a new shape 
function describing frontal width of the vegetation array; (2) new solutions of the velocity profile derived from momentum equations taking into account vegetation shape; and (3) new empirical expressions for parameters in solutions are provided. Comparison between modeled results and measured ones proves that the new solutions proposed here are effective for predicting velocity profiles in vegetated flows.

\section{Theory}

\subsection{Governing equations}

For steady, fully developed turbulent flow with vegetation, the flow region is divided into two layers: the vegetation layer and the surface layer (Fig. 1). In the Navier-Stokes momentum equation, the vegetation drag is considered as a body force in the vegetation layer. Choosing Cartesian coordinates, we define the streamwise direction as $x$, the transverse direction as $y$, and the vertical direction as $z$, with velocity components $\boldsymbol{V}=(u, v, w)$ corresponding to the coordinate directions $(x, y, z)$. Then, for a control volume, its governing equation is expressed in the streamwise direction as follows:

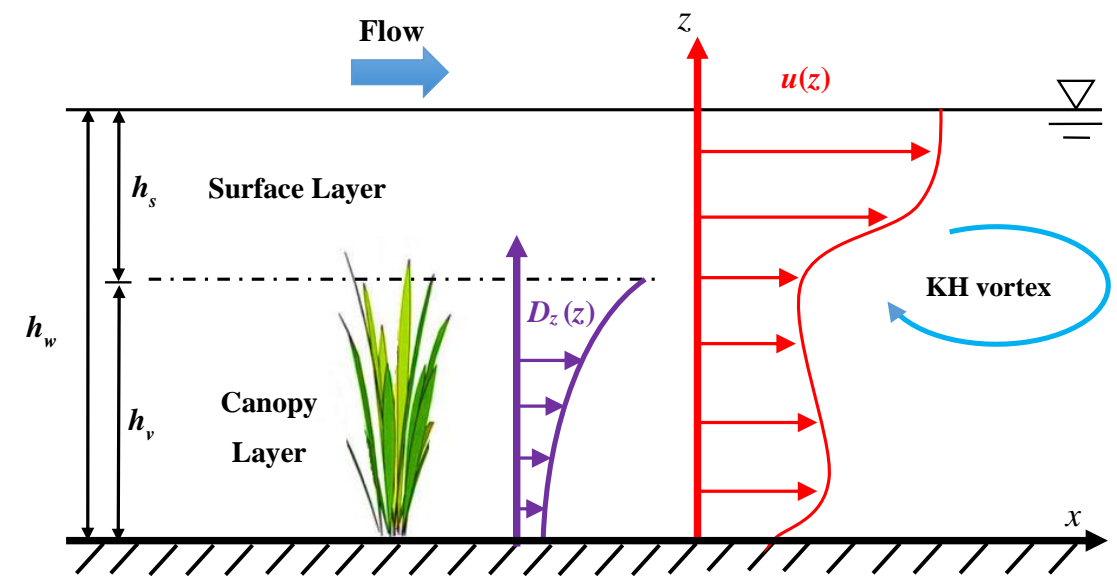

Figure 1: Sketch of flow through vegetation with non-uniform frontal width in the vertical direction 


$$
\frac{D u}{D t}=f_{x}-\frac{1}{\rho} \frac{\partial p}{\partial x}+\nu\left(\frac{\partial^{2} u}{\partial x^{2}}+\frac{\partial^{2} u}{\partial y^{2}}+\frac{\partial^{2} u}{\partial z^{2}}\right)
$$

where $f_{x}$ is the force acting on the control volume indicating the body force per unit mass, $p$ pressure, and $\nu$ kinematic viscosity.

The Reynolds-averaged approach applied to the above governing equation gives

$\frac{D \bar{u}}{D t}=\overline{f_{x}}-\frac{1}{\rho} \frac{\partial \bar{p}}{\partial x}+\nu\left(\frac{\partial^{2} \bar{u}}{\partial x^{2}}+\frac{\partial^{2} \bar{u}}{\partial y^{2}}+\frac{\partial^{2} \bar{u}}{\partial z^{2}}\right)+\left(\frac{\partial\left(-\overline{u^{\prime} u^{\prime}}\right)}{\partial x}+\frac{\partial\left(-\overline{u^{\prime} v^{\prime}}\right)}{\partial y}+\frac{\partial\left(-\overline{u^{\prime} w^{\prime}}\right)}{\partial z}\right)$,

which can be reduced to the following form when focusing on the streamwise direction

$$
\frac{D \bar{u}}{D t}=\overline{f_{x}}-\frac{1}{\rho} \frac{\partial \bar{p}}{\partial x}+\frac{1}{\rho} \frac{\partial \tau}{\partial z},
$$

where the total shear stress $\tau$, including viscous stress (which can always be ignored for turbulent flow) and Reynolds stress, is expressed as

$$
\tau=\rho \nu \frac{\partial \bar{u}}{\partial z}-\rho \overline{u^{\prime} w^{\prime}}
$$

with $-\rho \overline{u^{\prime} w^{\prime}}$ the Reynolds stress. The planar-averaged momentum equation for vegetated flow is then described as (Klopstra et al., 1996, Baptist et al., 2007. Liu et al., 2012, Huai et al., 2009, Poggi et al., 2009, Katul et al., 2011, Wang et al. 2015b)

$$
G_{x}+\delta F_{d}+\frac{1}{\rho} \frac{\partial \tau}{\partial z}=0
$$

where $G_{x}=g S_{o}$ is the gravitational component in the streamwise direction, $S_{o}$ the bed slope, parameter $\delta=-1$ denoting the vegetation layer and $\delta=0$ the surface layer, $F_{d}$ denotes the drag from the canopy acting on the control volume (different from the above mentioned $F_{d, b u l k}$ ) and is calculated using

$$
F_{d}=\frac{1}{2} C_{d} m D_{z}[u(z)]^{2}
$$

where $D_{z}$ is the frontal width of the vegetation stem (here the subscript $z$ signifies that this frontal width may vary with vertical direction $z$ ). 


\subsection{Models for shear stress}

To solve the momentum equation, the model for shear stress is the key component and has been investigated by many researchers. One should note that viscous stress can always be ignored for turbulent flow (Baptist et al. 2007, Luhar et al., 2008; Liu et al., 2012, Huai et al., 2014). Several models or theories have been proposed. They fall into two categories: non-differential and differential formulas.

\subsubsection{Non-differential formulas for shear stress}

For the non-differential formula, Shimizu et al. (1991) indicates that Reynolds stress follows an exponential profile for the vegetation layer, and this feature was confirmed in Dijkstra and Uittenbogaard (2010), in which the data displayed a similar trend with a peak value at the vegetation top. This trend was also validated in experiments for rigid (Huai et al. 2009) and flexible vegetation (Huai et al. 2013), and can be expressed in the form

$$
\tau_{v}=\tau_{\max } \exp \left[\alpha\left(z-h_{v}\right)\right]
$$

where subscript ' $v$ ' of the shear stress $\tau$ signifies the vegetation layer, $\alpha$ denotes a constant to be determined by the boundary conditions, and $\tau_{\max }=g S_{o} h_{s}$ is the peak value for shear stress at the top of the vegetation, $h_{s}=h_{w}-h_{v}$ being the height of the surface layer.

\subsubsection{Differential formula for shear stress}

For the differential formula of shear stress, the classic Boussinesqs eddy viscosity approach yields

$$
\tau=\rho \nu_{t} \frac{\partial u(z)}{\partial z}
$$

where $\nu_{t}$ is the eddy viscosity. Considered constant by Boussinesq in earlier years, a large body of experimental result indicate a variational behavior for eddy viscosities that depend on flow conditions. Theories for describing a variable eddy viscosity are summarized in the following: 
(i) Classic von Kármán-Prandtl mixing-length theory shows the eddy viscosity is linked with the velocity gradient as

$$
\nu_{t}=l^{2}\left|\frac{\partial u(z)}{\partial z}\right|,
$$

where the mixing lengths $l=\kappa z, \kappa=0.41$ is the Kármán constant, which is widely used in channel flow in the absence of vegetation. Further investigations (Katul et al., 2011, Poggi et al., 2004b a) showed that the mixing length is associated with the size of the vortex for different zones when vegetation is present. In the vegetation layer, the mixing length may be calculated using $l=2 \beta^{3} L_{c}$, where $\beta=u_{*} / u\left(h_{v}\right)$ is the coefficient of momentum absorption, and the friction velocity is $u_{*}=\sqrt{g S_{o} h_{s}}$. For the surface layer, the mixing length is described by $l=\kappa(z-d)$, where $d$ is the zero-plane displacement, which is defined by Thom (1971) adopting $d=\int_{0}^{h_{v}} \frac{d \tau}{d z} z d z / \int_{0}^{h_{v}} \frac{d \tau}{d z} d z$ and can be calculated using the formula from Katul et al. (2011) with $d=h_{v}-2 \beta^{3} L_{c} / \kappa$, where $L_{c}=\left(C_{d} a\right)^{-1}$ is the adjustment length scale (Belcher et al. 2003), indicating the loss of turbulent kinetic energy from advecting eddies due to vegetation (Katul et al., 2004), $a \approx L A I / h_{v}$ is the mean leaf area density, and $L A I$ the one-sided leaf area index.

(ii) Aside from the differential formula for eddy viscosity, several researchers have proposed that eddy viscosity is linked with the friction velocity times an adjusted length scale (Wang, 2012, Wang et al., 2015b)

$$
\nu_{t}=l u_{*} .
$$

or the velocity multiplied by an adjusted length scale (Baptist et al. 2007)

$$
\nu_{t}=\left(c_{p} l\right) u,
$$

${ }_{213}$ where $c_{p}$ is the intensity of turbulence calculated based on the turbulent kinetic energy $k_{t}$ and velocity $u(z)$. Further investigations have found that the product of turbulence $c_{p}$ and mixing length $l$ does not vary with vertical height $z$ (Liu et al. 2012, Van Velzen et al. 2003), expressed as a function of layer height

$$
c_{p} l=0.015 \sqrt{h_{v} h_{w}}
$$


217 and this expression was improved by Baptist et al. (2007), adopting the data of

${ }_{218}$ Nepf and Vivoni (2000) to obtain

$$
c_{p} l=\frac{h_{s}}{20} .
$$

222 were later tested.

223

229

230

which takes the form

In the following derivation, the eddy viscosity of type (ii) (equations 19 and 20) was adopted in the momentum equations in solving the velocity distribution.

The empirical equations for $c_{p} l$ from different research groups (equations 21 and 2.3. Velocity solution in the vegetation layer

Considering the solvability of the momentum equation, and with the adoption of equation 20, shear stress is expressed as

$$
\tau_{v}=c_{p} l \rho u(z) \frac{\partial u(z)}{\partial z} .
$$

226 Then the momentum equation gives

$$
\frac{1}{2} c_{p} l \frac{\partial^{2}[u(z)]^{2}}{\partial z^{2}}-\frac{1}{2} C_{d} m D_{z}[u(z)]^{2}+g S_{o}=0,
$$

$$
p_{1} \frac{\partial^{2}[u(z)]^{2}}{\partial z^{2}}+p_{2} D_{z}[u(z)]^{2}+p_{3}=0,
$$

228 with the introduction of parameters

$$
p_{1}=\frac{1}{2} c_{p} l
$$

$$
p_{2}=-\frac{1}{2} C_{d} m
$$

$$
p_{3}=g S_{o} .
$$

(i) For vegetation with uniform vertical width, $D_{z}=D_{0}$ is a constant, and the analytical solution of velocity profile gives

$$
u_{v}(z)=\sqrt{c_{1} \exp \left(\sqrt{-\frac{p_{2}}{p_{1}} D_{0}} z\right)+c_{2} \exp \left(-\sqrt{-\frac{p_{2}}{p_{1}} D_{0} z}\right)-\frac{p_{3}}{p_{2} D_{0}}} .
$$


252 and

(ii) For vegetation with width-varying shape, here we take shrub and sedge as representative (Liu et al., 2011, 2012, Huai et al., 2019), where the frontal width increases from bottom up (narrow at the bottom and wide near the top of the vegetation). Liu et al. (2011, 2012) proposed two shape functions to describe the effect of variable frontal width in flow the first being

$$
D_{z}=\frac{\left(1-2 n_{1}\right) D_{\max }}{4\left(n_{1} z / n_{2}+n_{3}\right)^{2}},
$$

and the second

$$
D_{z}=\left[\frac{n_{4} z+n_{5}}{n_{6} z^{2}+n_{7} z+n_{8}}\right]^{2} D_{\max },
$$

where $n_{i}(i=1,2,3 \ldots, 8)$ are parameters to be determined from vegetation morphotype and flow characteristics, and $D_{\max }$ is the maximum frontal width of vegetation. Three or five parameters are needed to build the shape of the vegetation array in these two approaches. From another point of view, these parameters include not only the physical attributes of the vegetation (frontal width), but also flow features such as turbulence intensity $C_{p}$, drag coefficient $C_{d}$, and mixing length scale $l$. Here, considering the solvability of the governing equation, a new shape function is proposed that only focuses on the physical shape of the vegetation. Requires just two parameters, we have

$$
D_{z}=\left(q_{1} z+q_{2}\right)^{-2},
$$

where $q_{1}$ and $q_{2}$ are determined by the physical shape of the vegetation. Assuming the vegetation gradually widens from bottom up, which is very common in nature (Liu et al. 2012), expressions for $q_{1}$ and $q_{2}$ linked with vegetation attributes were obtained,

$$
q_{1}=\frac{D_{\max }^{-1 / 2}-D_{\min }^{-1 / 2}}{h_{v}},
$$

$$
q_{2}=D_{\min }^{-1 / 2},
$$

where $D_{\min }$ is the minimum width at the bottom. 
Then the momentum equation (equation 25) with variable width (equation 32 was solved analytically to get the velocity distribution in the vegetation layer

$$
u_{v}(z)=\sqrt{c_{3}\left(q_{1} z+q_{2}\right)^{L_{1}}+c_{4}\left(q_{1} z+q_{2}\right)^{L_{2}}+c_{5}\left(q_{1} z+q_{2}\right)^{2}},
$$

where

$$
L_{1}=\frac{q_{1} \sqrt{p_{1}}+\sqrt{p_{1} q_{1}^{2}-4 p_{2}}}{2 q_{1} \sqrt{p_{1}}},
$$

where $c_{3}$ and $c_{4}$ are integration constants determined by the boundary conditions.

The parameters in the shape function also can be determined by boundary conditions. When $z=0$, the slip velocity is

$$
u_{\text {slip }}=\sqrt{c_{3}\left(q_{2}\right)^{L_{1}}+c_{4}\left(q_{2}\right)^{L_{2}}+c_{5}\left(q_{2}\right)^{2}}
$$

which is calculated from the momentum balance at the bottom of the channel

$$
u_{\text {slip }}=\sqrt{\frac{2 g S_{f}}{C_{d} m D_{\min }}},
$$

where $S_{f}$ is the energy slope.

From the momentum balance, the interfacial shear stress between the vegetation layer and the surface layer establishes another boundary condition at the top of the canopy (Klopstra et al. 1996, Wang et al., 2015b; Yang and Choi, 2010). When considering the shear stress in the vegetation layer at $z=h_{v}$,

$$
\left.\tau_{v}\right|_{z=h_{v}}=\left.\rho c_{p} l \cdot\left[u(z) \frac{\partial u(z)}{\partial z}\right]\right|_{z=h_{v}} .
$$

270 The shear stress in the surface layer at $z=h_{v}$ is

$$
\left.\tau_{s}\right|_{z=h_{v}}=\rho g h_{s} S_{f}
$$


Then, parameters $c_{3}$ and $c_{4}$ are obtained by solving the momentum equation combined with these two boundary conditions,

$c_{3}=-\frac{q_{2}^{L_{2}}\left[c_{5}\left(c_{p} l\right) q_{1}\left(h_{v} q_{1}+q_{2}\right)-g h_{s} S_{f}\right]-\frac{1}{2}\left(c_{p} l\right) L_{2} q_{1}\left(c_{5} q_{2}^{2}-u_{s l i p}^{2}\right)\left(h_{v} q_{1}+q_{2}\right)^{L_{2}-1}}{\frac{1}{2}\left(c_{p} l\right) L_{1} q_{1} q_{2}^{L_{2}}\left(h_{v} q_{1}+q_{2}\right)^{L_{1}-1}-\frac{1}{2}\left(c_{p} l\right) L_{2} q_{1} q_{2}^{L_{1}}\left(h_{v} q_{1}+q_{2}\right)^{L_{2}-1}}$,

$c_{4}=\frac{q_{2}^{L_{1}-L_{2}}\left\{q_{2}^{L_{2}}\left[c_{5}\left(c_{p} l\right) q_{1}\left(h_{v} q_{1}+q_{2}\right)-g h_{s} S_{f}\right]-\frac{1}{2}\left(c_{p} l\right) L_{2} q_{1}\left(c_{5} q_{2}^{2}-u_{s l i p}^{2}\right)\left(h_{v} q_{1}+q_{2}\right)^{L_{2}-1}\right\}}{\frac{1}{2}\left(c_{p} l\right) L_{1} q_{1} q_{2}^{L_{2}}\left(h_{v} q_{1}+q_{2}\right)^{L_{1}-1}-\frac{1}{2}\left(c_{p} l\right) L_{2} q_{1} q_{2}^{L_{1}}\left(h_{v} q_{1}+q_{2}\right)^{L_{2}-1}}$

$-\mathrm{q}_{2}^{-L_{2}}\left(c_{5} q_{2}^{2}-u_{\text {slip }}^{2}\right)$

\subsection{Velocity solution in the surface layer}

For flow in the surface layer $\left(z>h_{v}\right)$, the traditional method adopts the logarithmic function with

$$
u_{s}(z)=\frac{u_{*}}{\kappa} \ln \left(\frac{z-d}{z_{0}}\right)
$$

where two parameters are needed such as roughness height $z_{0}$ and zero-plane displacement $d$.

Here, we adopt another approach to predict velocity that needs only one parameter to shape the velocity profile. First, the momentum equation for surface layer gives

$$
\frac{\partial \tau_{s}}{\partial z}+\rho g S_{o}=0
$$

where the shear stress adopted equation 19 with mixing length $l=k_{n} z$, and $k_{n}$ is a coefficient determined by the flow characteristics and vegetation attributes

$$
\tau_{s}=\rho k_{n} u_{*} z \frac{\partial u(z)}{\partial z}
$$

where subscript ' $\mathrm{s}$ ' to the shear stress denotes the surface layer. Then, the analytical solution is obtained by substituting equation (47) into (46) yielding

$$
u_{s}(z)=-\frac{g S_{o}}{k_{n} u_{*}} z+c_{6} \ln z+c_{7}
$$

where parameters $c_{6}$ and $c_{7}$ are constants determined by the following two boundary conditions. 
Boundary condition (i): the velocity at the top of the canopy in the surface layer and the vegetation layer is the same

$$
u_{v}\left(h_{v}\right)=u_{s}\left(h_{v}\right)
$$

Boundary condition (ii): the velocity gradient is zero at the flow surface

$$
\left.\frac{\partial u(z)}{\partial z}\right|_{z=h_{w}}=0 .
$$

One obtains then parameters values

$$
c_{6}=\frac{g h_{w} S_{o}}{k_{n} u_{*}},
$$

$c_{7}=\frac{g S_{o}\left(h_{v}-h_{w} \ln h_{v}\right)+k_{n} u_{*} \sqrt{c_{3}\left(h_{v} q_{1}+q_{2}\right)^{L_{1}}+c_{4}\left(h_{v} q_{1}+q_{2}\right)^{L_{2}}+c_{5}\left(h_{v} q_{1}+q_{2}\right)^{2}}}{k_{n} u_{*}}$.

Next, the flow profile in the surface layer is described by equation (48). The approach in determining parameter $k_{n}$ is discussed in Section 4 .

\section{Experiments}

We conducted flume experiments in an open channel using modelled plants for simulating variable-width vegetation. Moreover, two set of experimental data from the literature were included to enrich the scope of the experimental data. Their details are described in the following.

\subsection{Present experiments}

Experiments were conducted in two glass channels at the State Key Laboratory of Water Resources and Hydropower Engineering Science in Wuhan University, China (details are given in Huai et al. (2019)). The size of these two channels were: $20 \mathrm{~m}$ long $\times 0.6 \mathrm{~m}$ wide $\times 0.4 \mathrm{~m}$ deep (bed slope $0.04 \%$ ) and $20 \mathrm{~m}$ long $\times 1.0 \mathrm{~m}$ wide $\times 0.4 \mathrm{~m}$ deep (bed slope $0.01 \%$ ). The channels were equipped with electro-magnetic flow meters for measuring flow discharge, and a tailgate located at the end of the channels to control flow depth. The vegetation was installed in a staggered arrangement along the bottom of the channel. The 
length of vegetation zone was set to $8 \mathrm{~m}$ to ensure a complete development of the flow within and above the vegetation.

For the simulated vegetation in this experiment, a meadow model plant was adopted to represent sedge. Each plant has 11 plastic slips and the diameter of the trunk was approximately $0.015 \mathrm{~m}$. The original averaged height of the sedge meadow model was $0.210 \mathrm{~m}$, and the lateral width ranged from $D_{\min }=0.02 \mathrm{~m}$ (at the bottom) to $D_{\max }=0.170 \mathrm{~m}$ (near the top). The artificial vegetation produced a small deflection because of its flexibility.

Velocity measurements were taken using an acoustic Doppler velocimeter. For model verification in Section 4 , the velocity profile at the location where $x / L_{v e g}>0.61$ was selected, at which point the flow reaches its fully developed condition (Huai et al., 2019).

\subsection{Liu et al.'s experiments}

Liu et al. (2011, 2012) conducted a series of experiments at the Hydraulic laboratory of Tsinghua University, China. They modeled shrub-like vegetation in an open channel $22.6 \mathrm{~m}$ long $\times 1.6 \mathrm{~m}$ wide $\times 0.8 \mathrm{~m}$ deep, with a bed slope of $0.67 \%$. The average height of the shrubs was $0.275 \mathrm{~m}$, with an increasing trend for the frontal width along the vertical direction from the bottom up. Here, $D_{\min }=0.05 \mathrm{~m}$ near the bottom (this value was not given in their publication but can be reasonably estimated from their photographs), and $D_{\max }=0.200 \mathrm{~m}$ near the top of the vegetation. The vegetation was fixed in a staggered arrangement with two distinct canopy densities: $m=15.71$ and 7.85 stems $/ \mathrm{m}^{2}$. The velocity profile was measured using an acoustic Doppler velocimeter.

\subsection{Nepf and Vivoni's experiments}

Nepf and Vivoni $(2000)$ conducted experiments with a $24 \mathrm{~m}$ long $\times 0.38 \mathrm{~m}$ wide recirculating glass wall flume. The model vegetation zone was $7.4 \mathrm{~m}$ long with a height of $0.16 \mathrm{~m}$, with a staggered arrangement of plants (vegetation density was $m=330$ stems $/ \mathrm{m}^{2}$ ). In addition, according to the principle proposed by Kouwen and Li (1980), their vegetation was constructed based on similarity 
Table 1: Summary of present research on flow in vegetation

\begin{tabular}{lcccccc}
\hline Data type & \multicolumn{3}{c}{ Present experiments } & \multicolumn{2}{c}{ Liu et al. } & Nepf and Vivoni \\
& Case 1 & Case 2 & Case 3 & Case 1 & Case 2 & Case 1 \\
\hline Flow depth, $h_{w}(\mathrm{~m})$ & 0.27 & 0.27 & 0.33 & 0.45 & 0.45 & 0.44 \\
Adjusted vegetation height, $h_{v}(\mathrm{~m})$ & 0.165 & 0.175 & 0.190 & 0.255 & 0.255 & 0.14 \\
Max width, $D_{\max }(\mathrm{m})$ & 0.17 & 0.17 & 0.17 & 0.2 & 0.2 & 0.017 \\
Min width, $D_{\min }(\mathrm{m})$ & 0.02 & 0.02 & 0.02 & 0.05 & 0.05 & 0.005 \\
Vegetation density, $m\left(\mathrm{stems} / \mathrm{m}^{2}\right)$ & 43.3 & 108.3 & 108.3 & 15.71 & 7.85 & 330 \\
Drag coefficient $C_{d}$ & 0.13 & 0.17 & 1.3 & 1.5 & 1.5 & 0.5 \\
Turbulence scale, $c_{p} l\left(\times 10^{-4} \mathrm{~m}\right)$ & 5 & 4.5 & 12 & 30 & 32 & 10 \\
Turbulence index, $k_{n}\left(\times 10^{-3}\right)$ & 40 & 40 & 50 & 50 & 45 & 60 \\
\hline
\end{tabular}

in geometry and flexural rigidity to prototype aquatic vegetation. Each plant consisted of six blades, each of width $0.003 \mathrm{~m}$. The morphology of a single plant featured a variable frontal width ranging from 0.005 to $0.017 \mathrm{~m}$.

\subsection{Summary of experimental parameters}

The experimental setup and vegetation attributes are listed in Table 1. As there were waves near the top of the vegetation resulting in a greatly reduced resistance at this point, an adjusted vegetation height was proposed where the height was slightly lower $(0.02 \mathrm{~m})$ than the reported vegetation height. Morepver, note that some parameters, such as flow depth of the experiments of Liu et al. (2012)'s were not given and hence we estimated values from velocity profiles of their paper (Although there may be minor errors, such errors would have little effect on the final calculation).

\section{Results and discussion}

This section is divided into three subsections: (1) A comparison of the analytical velocity solutions for the whole depth with measured data is discussed in Section 4.1. (2) Features of the drag coefficient are discussed in Section 4.2 and (3) turbulence features are discussed in Section 4.3 


\subsection{Velocity profile comparisons}

A comparison between the analytical solutions of the velocity profile (adopting best-fitted parameters, which will be discussed later) and measured data is shown in Fig. 2 The solution proposed here is seen to reproduce well the velocity profile for flow through vegetation with variable frontal width. The following subsections present the procedure used in deriving the empirical expressions for the adjusted parameters.
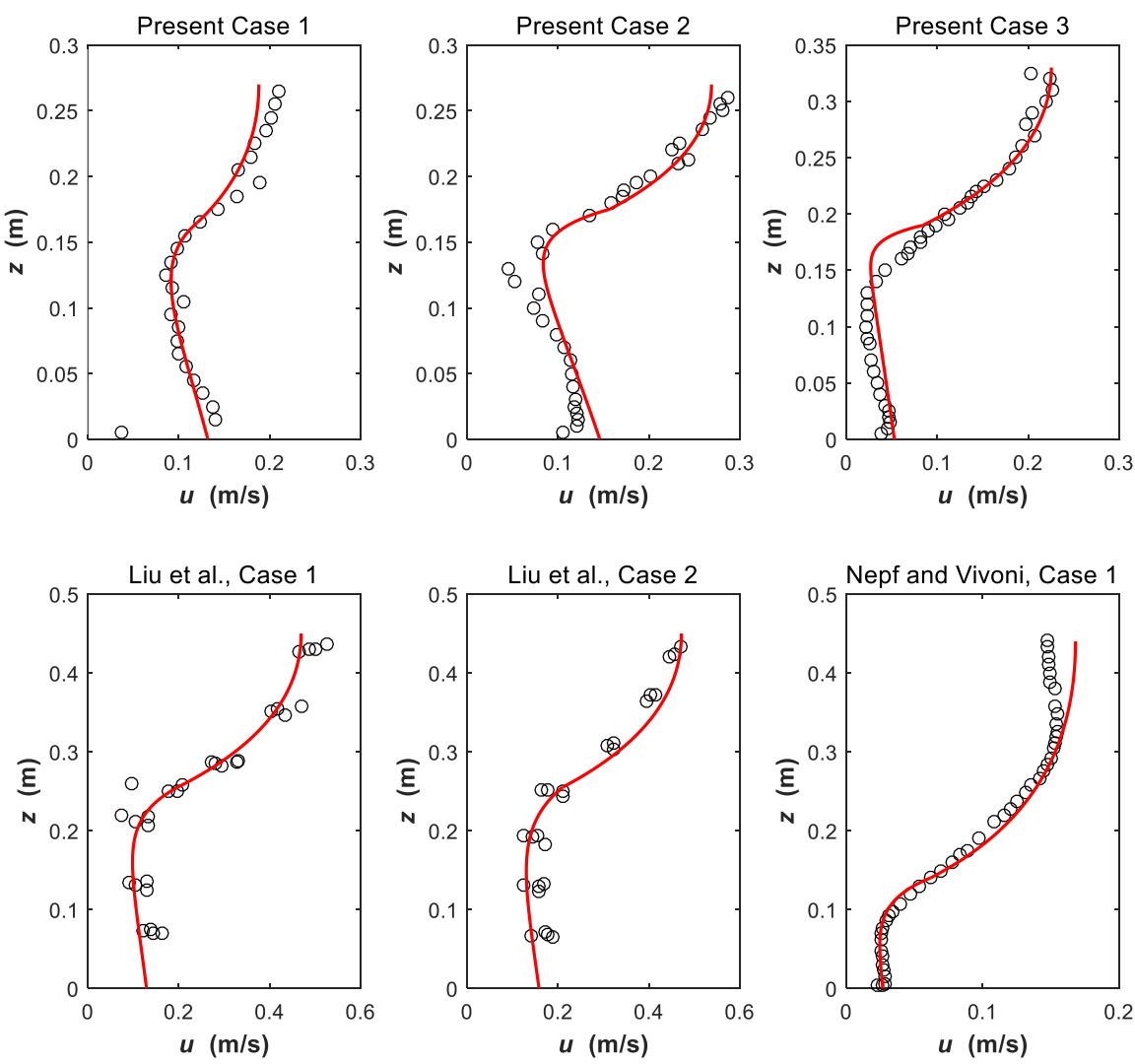

Figure 2: Comparison of analytical solutions with measured data for different cases 


\subsection{Features of the drag coefficient}

Drag coefficient $C_{d}$ is a key factor for quantifying the resistance to flow (Nepf and Ghisalberti, 2008; Wang et al., 2018a; Baptist et al., 2007). For an isolated cylinder, the local $C_{d}$ can be calculated from (Cheng, 2012)

$$
C_{d, i s o}=11 R e_{d}^{-0.75}+0.9 \Gamma_{1}\left(R e_{d}\right)+1.2 \Gamma_{2}\left(R e_{d}\right)
$$

where

$$
\Gamma_{1}\left(R e_{d}\right)=1-\exp \left(-\frac{1000}{R e_{d}}\right)
$$

and

$$
\Gamma_{2}\left(R e_{d}\right)=1-\exp \left[-\left(\frac{R e_{d}}{4500}\right)^{0.7}\right],
$$

where the Reynolds number for a cylinder is $R e_{d}=U d / \nu$ with $U$ the approaching velocity. However, for flow through a vegetation array ('array' means more than just a single stem in the flow), resistance mechanisms become complicated, and the $C_{d}$ of a vegetated array differs much from an isolated stem $\left(C_{d, i s o}\right)$. When only considering the effect of vegetation density on the drag coefficient, $C_{d}$ displays an increasing trend (Tanino and Nepf, 2008; Stoesser et al., 2010) or a decreasing trend (Nepf, 1999; Lee et al., 2004) when increasing the vegetation density (Etminan et al. 2017). This indicates that the drag due to the vegetation array cannot be assessed by only the vegetation density. Hence a vegetation-related Reynolds number is introduced, $R e_{v}=\left(R_{v} / D\right) R e_{d}$. Many experiments show a monotonic decline in $C_{d}$ with increasing vegetation-related Reynolds number $\left(R e_{v}\right)$ for canopies composed of cylinders (Ishikawa et al. 2000, James et al., 2004, Tanino and Nepf, 2008; Liu et al., 2008; Ferreira et al. 2009 Kothyari et al. 2009 Stoesser et al. 2010), which can be expressed as (Wang et al., 2019)

$$
C_{d, w a n g}=0.819+\frac{58.5}{\sqrt{\frac{\pi(1-\phi)}{4 \phi} R e_{d}}} .
$$

Due to the variation of $D_{z}$ in the vertical direction, there is a need to calculate the averaged width $D_{\text {ave }}$ when adopting these formulas (equations 53 and 
385

56). Based on shape function (equation 32), the averaged width is calculated from

$$
D_{\text {ave }}=\frac{1}{q_{2}\left(q_{1} h_{v}+q_{2}\right)} .
$$

Then, the Reynolds number for vegetation with variable frontal width reads

$$
R e_{d}=\frac{U_{v} D_{a v e}}{\nu},
$$

where $U_{v}$ is the averaged velocity for the vegetation layer.

Although the above formula of $C_{d}$ was mainly derived based on cylinder or strip with constant width $D$, the left and middle panels in Fig. 3 illustrate that the predicted $C_{d}$ using equations (53) and (56) deviates from the measured value, and shows that they are not suitable for vegetation with variable $D_{z}$, where the measured $C_{d}$ is obtained by matching the calculated velocity profile to the measured value. Hence the drag coefficient $C_{d}$ here needs to reflect the above-mentioned vegetation shape with $D_{\min }$ and $D_{\max }$. The following formula was proposed by incorporating the traditional Reynolds number in with the plant morphology descriptors

$$
C_{d, \text { new }}=\frac{R e_{d}}{1110}\left(\frac{2 D_{\min }-D_{a v e}}{D_{\text {ave }}}\right)+\left(\frac{51.4 D_{a v e}-9.85 D_{\max }-60.5 D_{\min }}{D_{a v e}}\right) .
$$

8 A comparison between measured and the newly derived empirical drag coefficient (right panel of figure 3) show good agreement proving that equation 59 matches the measured value very well.

\subsection{Turbulence features}

\subsubsection{Vegetation layer}

The turbulence scale $c_{p} l$ derived in previous studies on vegetation flow with cylinders or stripes shows a dependence on layer height (equations 21 and 22). However, comparisons between the measured $C_{p} l$ with values from these two equations show that they are not suitable for vegetation of distinct shape (see left and middle panels of Fig. 4). Based on the height of each layer, a new 

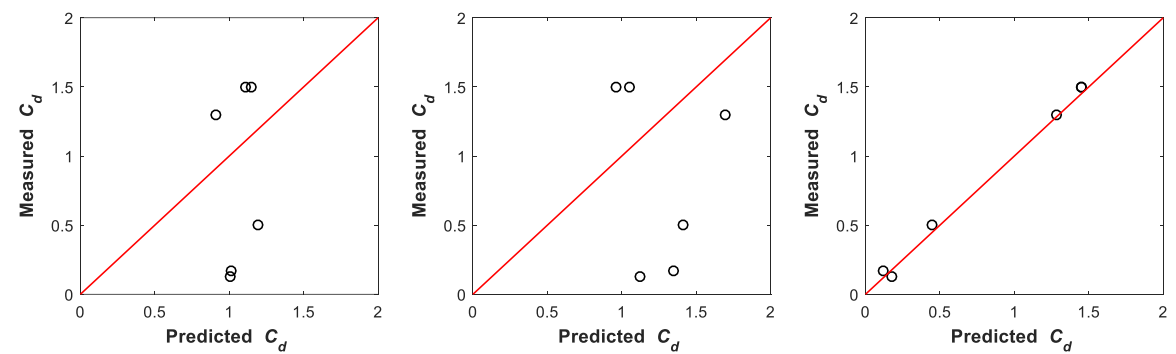

Figure 3: Comparison between measured and predicted drag coefficients. The predicted $C_{d} \mathrm{~s}$ given in the left, middle, and right panels are $C_{d, i s o}$ from equation $53, C_{d, \text { wang }}$ from equation [56, and $C_{d, \text { new }}$ from equation 59 , respectively.
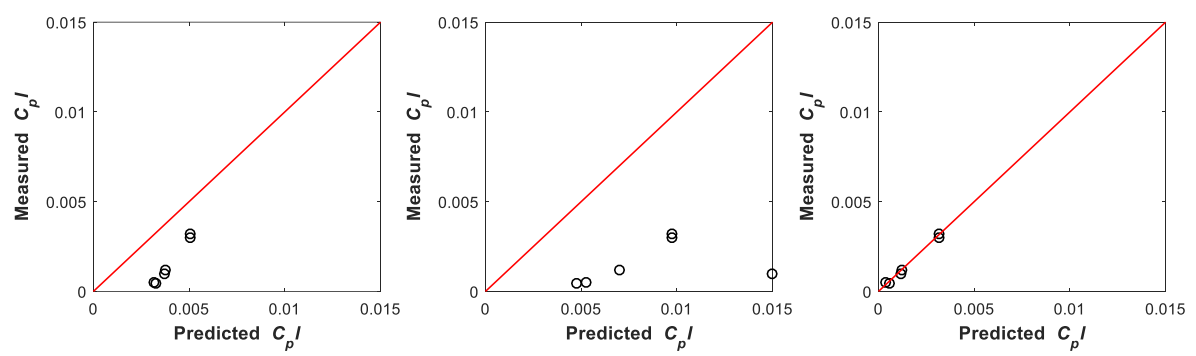

Figure 4: Comparison between measured and predicted formula for turbulence length scale $c_{p} l$ in the vegetation layer. The predicted $C_{p} l$ for the left, middle, and right panels are obtained from equations 21 and 22 , and proposed equation 60 , respectively.

empirical expression is proposed for the vegetation with variable frontal width, specifically

$$
c_{p} l=\frac{1}{42} h_{w}-\frac{1}{60} h_{s}-\frac{1}{233} .
$$

A comparison (right panel of Fig. 4) shows that this formula is in good agreement with the measured turbulence length scale for the instance discussed here.

\subsubsection{Surface layer}

The traditional logarithmic formula (equation 45) describes the flow profile of the surface layer with two parameters: zero-plane displacement $d$ and hydrodynamic roughness height $z_{0}$. We now adopt another approach which only 


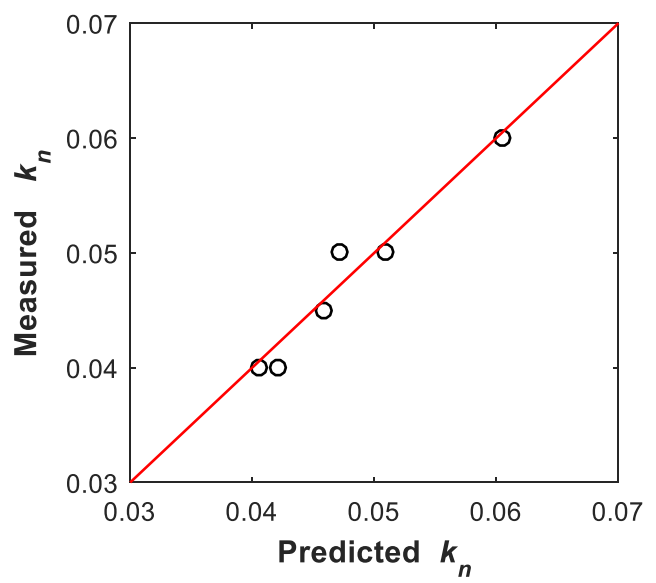

Figure 5: Comparison between measured and predicted turbulence index $k_{n}$ in surface layer.

needs one parameter $k_{n}$ for shaping the velocity profile (equation 48). This $k_{n}$ is seen as a combination of $d$ and $z_{0}$ but is complicated and influenced by many factors. Here, for simplicity, dimensionless $h_{v} / L_{c}$ (including the vegetation attribute and resistance length scale of vegetation) and the ratio of layer height are taken as influence factors, yielding the following fitted expression

$$
k_{n}=\frac{2}{25}+\frac{1}{310} \frac{h_{v}}{L_{c}} \frac{h_{v}}{h_{s}}-\frac{1}{16} \frac{h_{v}}{h_{w}} .
$$

A comparison between measured and predicted $k_{n}$ is shown in Fig. 5 , indicating that the proposed formula well matches the turbulence index $k_{n}$ for instances reported above.

\subsection{Limitations}

Although the shape function proposed in equation (32) illustrates a gradual increasing trend in frontal width $D_{z}$ from bottom up and is suitable for vegetation such as shrub and sedge, the investigation needs to be broadened to other types of vegetation. Moreover, although empirical expressions for the three parameters $C_{d}, c_{p} l$, and $k_{n}$ were proposed for various instances studied here, 
further investigation is still needed in considering different vegetation shapes and flow conditions.

\section{Conclusion}

Different from former analytical models of velocity distribution in literature, which is mainly suitable for vegetation stems with uniform frontal width, this study established new analytical solutions of the velocity profile for vegetation with shape. A brief summary of results is presented in the following:

(1) Vegetation such as shrub and sedge of certain shape where the frontal width $D_{z}$ displays a gradual increasing trend with vertical direction from the bottom up was investigated. A new vegetation shape function describing the frontal width $D_{z}$ was proposed linked with $D_{\max }$ and $D_{\min }$ and needs fewer parameters than previous formulas.

(2) Different closure models for eddy viscosity were adopted for the twolayer vegetated flow, where eddy viscosity was expressed as a product of velocity scaled by an adjusted length scale in the vegetation layer and the friction velocity scaled by an adjusted length scale in surface layer. Combined with the proposed shape function describing frontal width of vegetation, analytical solutions of the velocity profile were derived from the momentum equations. Good agreement between calculated and measured data shows the analytical model is effective for predicting velocity profile.

(3) Because the shape of vegetation is different from the simplified vegetation models using cylinders or single strips, the former empirical formulas of the drag coefficient and the turbulence length scale were not suitable for the widthvarying vegetation here. New empirical expression were proposed that matched the observed data well.

(4) There were few experiments focused on width-varying vegetation up to now. Although empirical expressions for determining parameters such as drag coefficient $C_{d}$, turbulence length scale for vegetation layer $c_{p} l$, and surface layer $k_{n}$ were proposed for a number of limited scenarios, further investigation and 
459 more experiments are needed in considering different vegetation types and flow 460 conditions. 


\section{${ }_{461}$ Acknowledgments}

${ }_{462}$ The authors acknowledge support through the following projects: National

${ }_{463}$ Natural Science Foundation of China (51809286, 51439007, 11672213, and 11872285);

${ }_{464}$ Research and Development Support Program of China Institute of Water Re-

465 sources and Hydropower Research (WE0145B062019); and China Postdoctoral

466 Science Foundation (2017M610949 and 2018T110122). 


\section{References}

Baptist, M., Babovic, V., Rodríguez Uthurburu, J., Keijzer, M., Uittenbogaard, R., Mynett, A., Verwey, A., 2007. On inducing equations for vegetation resistance. Journal of Hydraulic Research 45, 435-450. doi:10.1080/00221686. 2007.9521778 .

Belcher, S.E., JERRAM, N., HUNT, J.C.R., 2003. Adjustment of a turbulent boundary layer to a canopy of roughness elements. Journal of Fluid Mechanics 488, 369-398.

Cheng, N.S., 2012. Calculation of drag coefficient for arrays of emergent circular cylinders with pseudofluid model. Journal of Hydraulic Engineering 139, 602611. doi 10.1061/(ASCE)HY.1943-7900.0000722.

Cheng, N.S., 2015. Single-layer model for average flow velocity with submerged 1 rigid cylinders. Journal of Hydraulic Engineering doi 10.1061/(ASCE)HY. 1943-7900.0001037.

Constança Aguiar, F., Rosário Fernandes, M., Teresa Ferreira, M., 2011. Riparian vegetation metrics as tools for guiding ecological restoration in river-

口 scapes. Knowl. Managt. Aquatic Ecosyst. , 21URL: https://doi.org/10. $1051 / \mathrm{kmae} / 2011074$, doi $10.1051 / \mathrm{kmae} / 2011074$.

Dijkstra, J., Uittenbogaard, R., 2010. Modeling the interaction between flow 口 and highly flexible aquatic vegetation. Water Resources Research 46. doi 10 . 1029/2010WR009246.

Etminan, V., Lowe, R.J., Ghisalberti, M., 2017. A new model for predicting the drag exerted by vegetation canopies. Water Resources Research 53, 3179-3196. URL: http://dx.doi.org/10.1002/2016WR020090, doi $10.1002 / 2016$ WR020090.

Ferreira, R.M., Ricardo, A.M., Franca, M.J., 2009. Discussion of "laboratory investigation of mean drag in a random array of rigid, emergent cylinders" 
by yukie tanino and heidi m. nepf. Journal of Hydraulic Engineering 135, 690-693. doi:10.1061/(ASCE) HY.1943-7900.0000021.

Ghisalberti, M., Nepf, H.M., 2002. Mixing layers and coherent structures in n vegetated aquatic flows. Journal of Geophysical Research 107, 3011. doi 10. 1029/2001JC000871.

Huai, W.X., Wang, W.J., Hu, Y., Zeng, Y.H., Yang, Z.H., 2014. Analytical model of the mean velocity distribution in an open channel with doublelayered rigid vegetation. Advances in Water Resources 69, 106-113. doi doi. org $/ 10.1016 / j$.advwatres.2014.04.001.

Huai, W.X., Wang, W.J., Zeng, Y.H., 2013. Two-layer model for open channel flow with submerged flexible vegetation. Journal of Hydraulic Research 51, 708-718. doi:10.1080/00221686.2013.818585.

Huai, W.X., Zeng, Y.H., Xu, Z.G., Yang, Z.H., 2009. Three-layer model for vertical velocity distribution in open channel flow with submerged rigid vegetation. Advances in Water Resources 32, 487-492. doi:10.1016/j.advwatres. 2008.11.014.

Huai, W.X., Zhang, J., Katul, G., Cheng, Y.G., Tang, X., Wang, W.J., 2019. The structure of turbulent flow through submerged flexible vegetation. Journal of Hydrodynamics 31, 774-781. doi:10.1007/s42241-019-0023-3.

Ishikawa, Y., Mizuhara, K., Ashida, S., 2000. Effect of density of trees on drag exerted on trees in river channels. Journal of Forest Research 5, 271-279. doi:10.1007/BF02767121.

James, C., Birkhead, A., Jordanova, A., O'sullivan, J., 2004. Flow resistance of emergent vegetation. Journal of Hydraulic Research 42, 390-398. doi:10. $1080 / 00221686.2004 .9728404$.

Katul, G.G., Mahrt, L., Poggi, D., Sanz, C., 2004. One-and two-equation models for canopy turbulence. Boundary-Layer Meteorology 113, 81-109. 
Katul, G.G., Poggi, D., Ridolfi, L., 2011. A flow resistance model for assessing the impact of vegetation on flood routing mechanics. Water Resources Research 47. doi:10.1029/2010WR010278

Katul, G.G., Wiberg, P., Albertson, J., Hornberger, G., 2002. A mixing layer theory for flow resistance in shallow streams. Water Resources Research 38. doi:10.1029/2001WR000817.

Klopstra, D., Barneveld, H., Van Noortwijk, J., Van Velzen, E., 1996. Analytical model for hydraulic roughness of submerged vegetation, in: 27th IAHR Congress, HKV Consultants. pp. 775-780.

Kothyari, U.C., Hayashi, K., Hashimoto, H., 2009. Drag coefficient of unsubmerged rigid vegetation stems in open channel flows. Journal of Hydraulic Research 47,691-699. doi 10.3826/jhr.2009.3283.

Kouwen, N., Li, R., 1980. Biomechanics of vegetative channel linings. J. Hydraul. Div 106, 1085-1103.

Kouwen, N., Unny, T., Hill, H.M., 1969. Flow retardance in vegetated channels. Journal of the Irrigation and Drainage Division 95, 329-344.

Lee, J., Roig, L., Jenter, H., Visser, H., 2004. Drag coefficients for modeling flow through emergent vegetation in the florida everglades. Ecological Engineering 22, 237-248. doi $10.1016 / \mathrm{j}$.ecoleng.2004.05.001.

Li, Z., Liu, X., Niu, T., Zhou, Q., Ma, T., Gao, Y., 2015. Ecological restoration and its effects on a regional climate: The source region of the yellow river, china. Environmental Science Technology 49, 5897.

Liu, D., Diplas, P., Fairbanks, J.D., Hodges, C.C., 2008. An experimental study of flow through rigid vegetation. Journal of Geophysical Research: Earth Sur-

face 113. URL: http://dx.doi.org/10.1029/2008JF001042, doi 10.1029/ 2008JF001042. 
Liu, D., Diplas, P., Hodges, C.C., Fairbanks, J.D., 2010. Hydrodynamics of flow through double layer rigid vegetation. Geomorphology 116, 286-296.

Liu, Z.W., Chen, Y.C., Zhu, D.J., Hui, E.Q., Jiang, C.B., 2011. Vertical profile of horizontal velocity of flow through shrubs (in chinese). Journal of Hydroelectric Engineering 30, 237-241.

Liu, Z.W., Chen, Y.C., Zhu, D.J., Hui, E.Q., Jiang, C.B., 2012. Analytical model for vertical velocity profiles in flows with submerged shrub-like vegetation. Environmental Fluid Mechanics 12, 341-346. doi 10.1007/ s10652-012-9243-6.

Lu, J., Dai, H.C., 2016. Large eddy simulation of flow and mass exchange in an embayment with or without vegetation. Applied Mathematical Modelling 40, 7751-7767.

Luhar, M., Rominger, J., Nepf, H.M., 2008. Interaction between flow, transport and vegetation spatial structure. Environmental Fluid Mechanics 8, 423-439.

Mi, Y., He, C., Bian, H., Cai, Y., Sheng, L., Liang, M., 2015. Ecological engineering restoration of a non-point source polluted river in northern china. Ecological Engineering 76, 142-150.

Nepf, H.M., 1999. Drag, turbulence, and diffusion in flow through emergent vegetation. Water Resources Research 35, 479-489. doi:10.1029/1998WR900069

Nepf, H.M., 2012. Flow and transport in regions with aquatic vegetation. Annual Review of Fluid Mechanics 44, 123-142. doi 10.1146/ annurev-fluid-120710-101048

Nepf, H.M., Ghisalberti, M., 2008. Flow and transport in channels with submerged vegetation. Acta Geophysica 56, 753-777. doi 10.2478/ s11600-008-0017-y.

Nepf, H.M., Vivoni, E.R., 2000. Flow structure in depth-limited, vegetated flow. Journal of Geophysical Research: Oceans 105, 28547-28557. doi 10 . 1029/2000JC900145. 
Okamoto, T.A., Nezu, I., 2009. Turbulence structure and monami phenomena in flexible vegetated open-channel flows. Journal of Hydraulic Research 47, 798-810.

Peters, T., 2012. Turbulence, coherent structures, dynamical systems and symmetry, 2nd edn., by p. holmes. Contemporary Physics 53, 1-1.

Poggi, D., Katul, G., Albertson, J., 2004a. Momentum transfer and turbulent kinetic energy budgets within a dense model canopy. Boundary-Layer Meteorology 111, 589-614. doi:10.1023/B:BOUN.0000016502.52590.af.

Poggi, D., Krug, C., Katul, G.G., 2009. Hydraulic resistance of submerged rigid vegetation derived from first-order closure models. Water Resources Research 45. doi:10.1029/2008WR007373.

Poggi, D., Porporato, A., Ridolfi, L., Albertson, J., Katul, G., 2004b. The effect of vegetation density on canopy sub-layer turbulence. Boundary-Layer Meteorology 111, 565-587. doi:10.1023/B:BOUN.0000016576.05621.73.

Shimizu, Y., Tsujimoto, T., Nakagawa, H., Kitamura, T., 1991. Experimental study on flow over rigid vegetation simulated by cylinders with equi-spacing, in: Proceedings of the Japan Society of Civil Engineers, pp. 31-40.

Stoesser, T., Kim, S., Diplas, P., 2010. Turbulent flow through idealized emergent vegetation. Journal of Hydraulic Engineering 136, 1003-1017. doi:10.1061/(ASCE) HY.1943-7900.0000153.

Stoesser, T., Salvador, G.P., Rodi, W., Diplas, P., Shavit, U., 2009. Large eddy simulation of turbulent flow through submerged vegetation. Transport in Porous Media 78, 347-365.

Stromberg, J.C., 2001. Restoration of riparian vegetation in the south-western united states: importance of flow regimes and fluvial dynamism. J Arid Ent 49, 17-34. 
Tanino, Y., Nepf, H.M., 2008. Laboratory investigation of mean drag in a random array of rigid, emergent cylinders. Journal of Hydraulic Engineering 134, 34-41. doi 10.1061/(ASCE) 0733-9429(2008)134:1(34)

Thom, A., 1971. Momentum absorption by vegetation. Quarterly Journal of the Royal Meteorological Society 97, 414-428.

Thompson, S., Katul, G., Konings, A., Ridolfi, L., 2011. Unsteady overland flow on flat surfaces induced by spatial permeability contrasts. Advances in Water Resources 34, 1049-1058. doi:10.1016/j.advwatres.2011.05.012

Van Velzen, E., Jesse, P., Cornelissen, P., Coops, H., 2003. Hydraulic resistance of vegetation in floodplains, part 2: Background document version 1-2003. Ministry of Transport, Public Works and Water Management, Institute for Inland Water Management and Waste Water Treatment, report .

Wang, W., 2012. An analytical model for mean wind profiles in sparse canopies. Boundary-Layer Meteorology 142, 383-399.

Wang, W.J., Huai, W.X., Thompson, S., Katul, G.G., 2015a. Steady nonuniform shallow flow within emergent vegetation. Water Resources Research 51, 10047-10064. doi:10.1002/2015WR017658.

Wang, W.J., Huai, W.X., Thompson, S., Peng, W.Q., Katul, G.G., 2018a. Drag coefficient estimation using flume experiments in shallow non-uniform water flow within emergent vegetation during rainfall. Ecological Indicators 92, 367-378. doi:10.1016/j.ecolind.2017.06.041

Wang, W.J., Huai, W.X., Zeng, Y.H., Zhou, J.F., 2015b. Analytical solution of velocity distribution for flow through submerged large deflection flexible vegetation. Applied Mathematics and Mechanics 36, 107-120. doi 10.1007/ s10483-015-1897-9.

Wang, W.J., Peng, W.Q., Huai, W.X., Katul, G., Liu, X.B., Dong, F., Qu, X.D., Zhang, H.P., 2018b. Derivation of canopy resistance in turbulent flow from first-order closure models. Water 10, 1782. doi 10.3390/w10121782 
Wang, W.J., Peng, W.Q., Huai, W.X., Katul, G., Liu, X.B., Qu, X.D., Dong, F., 2019. Friction factor for turbulent open channel flow covered by vegetation. Scientific Reports , 5178.

Wang, W.J., Peng, W.Q., Huai, W.X., Qu, X.D., Dong, F., Feng, J., 2018c. Roughness height of submerged vegetation in flow based on spatial structure. Journal of Hydrodynamics 30, 754-757. doi 10.1007/s42241-018-0060-3.

Yang, W., Choi, S.U., 2010. A two-layer approach for depth-limited openchannel flows with submerged vegetation. Journal of Hydraulic Research 48, 466-475. doi:10.1080/00221686.2010.491649

Yu, S., Wang, M., 2014. Comprehensive evaluation of scenario schemes for multi-objective decision-making in river ecological restoration by artificially recharging river. Water Resources Management 28, 5555-5571.

Zhang, M., Hao, Z., Zhang, Y., Wu, W., 2013. Numerical simulation of solitary and random wave propagation through vegetation based on vof method. Acta Oceanologica Sinica 32, 38-46. 